\title{
Effects of thermal fluctuations in the SPOrt experiment
}

\author{
E. Carretti ${ }^{1}$, M. Zannoni ${ }^{2}$, C. Macculi ${ }^{1}$, S. Cortiglioni ${ }^{1}$, and C. Sbarra ${ }^{1}$ \\ 1 IASF/CNR Bologna, via Gobetti 101, 40129 Bologna, Italy \\ e-mail: carretti@bo.iasf.cnr.it \\ 2 IASF/CNR Milano, via Bassini 15, 20133 Milano, Italy
}

Received 29 April 2004 / Accepted 20 August 2004

\begin{abstract}
The role of systematic errors induced by thermal fluctuations is analyzed for the SPOrt experiment with the aim at estimating their impact on the measurement of the Cosmic Microwave Background Polarization (CMBP). The transfer functions of the antenna devices from temperature to data fluctuations are computed, by writing them in terms of both instrument and thermal environment parameters. In addition, the corresponding contamination maps are estimated, along with their polarized power spectra, for different behaviours of the instabilities. The result is that thermal effects are at a negligible level even for fluctuations correlated with the Sun illumination provided their frequency $f_{\mathrm{tf}}$ is larger than that of the Sun illumination $\left(f_{\text {day }}\right)$ by a factor $f_{\mathrm{tf}} / f_{\text {day }}>30$, which defines a requirement for the statistical properties of the temperature behaviour. The analysis with actual SPOrt operative parameters shows that the instrument is only weakly sensitive to temperature instabilities, the main contribution coming from the cryogenic stage. The contamination on the $E$-mode spectrum does not significantly pollute the CMBP signal and no specific data cleaning seems to be needed.
\end{abstract}

Key words. polarization - cosmology: cosmic microwave background - instrumentation: polarimeters - methods: data analysis

\section{Introduction}

The tiny level of the Cosmic Microwave Background Polarization (CMBP) requires a careful understanding and estimate of all systematic effects, which, if not kept under control, can jeopardize the measurement of the signal.

In the last years several works have been published on this topic, witnessing an increasing interest in systematics (e.g. Piat et al. 2000; Carretti et al. 2001; Leahy et al. 2002; Kaplan \& Delabrouille 2002; Mennella et al. 2002; Hu et al. 2003; Page et al. 2003; Franco et al. 2003; Carretti et al. 2004). Among others, thermal fluctuations can seriously affect the data and many teams have studied the impact on their CMB experiments (e.g. Piat et al. 2000; Mennella et al. 2002; Page et al. 2003). The importance of thermal fluctuations depends on the receiver scheme and, in some cases, can be crucial. An example is the total power architecture, where the variations induced onto the data are only slightly dumped with respect to the primary temperature fluctuations, making the detection of a $\mu \mathrm{K}$ signal a real challenge even in a very stable thermal environment. Thus, besides a quiet environment, receivers with a low sensitivity to temperature are necessary to weaken thermal fluctuations effects. Having a low offset generation, correlators minimize the thermal disturbance.

In this work we present an estimate of the thermal contamination for the SPOrt ${ }^{1}$ experiment (Cortiglioni et al. 2004), a set of four correlation radio-polarimeters devoted to measure the

\footnotetext{
${ }^{1}$ http://sport.bo.iasf.cnr.it
}

Stokes parameters $Q$ and $U$ of CMBP with an angular resolution of $F W H M=7^{\circ}$ from the International Space Station (ISS). The ISS environment is not optimal from the thermal point of view. In fact, thermal simulations show that the Sun illumination modulation due to the motion around the Earth induces orbit-synchronous temperature fluctuations with $\sim 3 \mathrm{~K}$ amplitude in case no active temperature control is used. Thus, a careful analysis of thermal effects is mandatory.

In the following we derive the transfer functions from temperature to data fluctuations to evaluate the sensitivity of the SPOrt radiometers to thermal instabilities. In addition, we study the contamination on the $E$-mode signal as a function of the statistical properties of the temperature instabilities, aiming at estimating its impact on the cosmological signal.

We show that the SPOrt correlation receivers are naturally an optimal architecture to keep under control the noise induced by temperature variations, thanks to their low sensitivity to temperature. In fact, the contamination on the $E$-mode is close to the cosmological signal already in case of free temperature fluctuations without thermal control, even though not sufficiently low to allow a clean detection. The adoption of an active control for the horn section further reduces the spurious signal at a comfortably negligible level, leaving the CMBP signal uncontaminated.

The paper is organized as follows: the computation of the transfer functions is presented in Sect. 2, whereas the effects of the fluctuation statistics and the SPOrt scanning strategy are presented in Sect. 3. The transfer functions and the 
contamination on the $E$-mode specific to the SPOrt experiment are given in Sect. 4 and, finally, the conclusions are summarized in Sect. 5.

\section{Transfer functions}

Thermal fluctuation effects can be evaluated starting from the offset generation equation. In SPOrt-like correlation polarimeters the offset is mainly generated by horn, polarizer and orthomode transducer (OMT), according to the equation ${ }^{2}$ (Carretti et al. 2001)

$$
\begin{aligned}
Q+j U= & S P_{\text {omt }}\left(T_{\text {sky }}+T_{\text {atm }}+T_{\text {noise }}^{\text {Ant }}\right) \\
& +S P_{\text {pol }}\left(T_{\text {sky }}+T_{\text {atm }}+T_{\text {noise }}^{\mathrm{h}}-A_{\mathrm{h}} T_{\mathrm{ph}}^{\mathrm{p}}\right),
\end{aligned}
$$

where $S P_{\text {omt }}$ and $S P_{\text {pol }}$ are

$S P_{\text {omt }}=A_{\text {omt }}\left(S_{A 1} S_{B 1}^{*}+S_{A 2} S_{B 2}^{*}\right)$,

$S P_{\mathrm{pol}}=\frac{1}{2}\left(1-\frac{A_{\|}}{A_{\perp}}\right)=\frac{1}{2} \frac{A_{\perp}-A_{\|}}{A_{\perp}}$,

$S_{A 1}, S_{B 2}$ the transmission parameters of the two OMT arms, $S_{A 2}, S_{B 1}$ their isolation terms, $A_{\mathrm{h}}, A_{\text {omt }}$ the attenuations of the horn and the OMT, respectively, and $A_{\perp}, A_{\|}$the attenuations of the polarizer along its two main polarizations. The offset sources are thus the physical temperature of the polarizer $T_{\mathrm{ph}}^{\mathrm{p}}$ and the signals propagating in the antenna system: the signal collected from the sky ( $T_{\text {sky }}$, in antenna temperature), the atmosphere emission $\left(T_{\mathrm{atm}}\right)$ and the noise temperatures of the horn alone $\left(T_{\text {noise }}^{\mathrm{h}}\right)$ and of the whole antenna system

$$
T_{\text {noise }}^{\text {Ant }}=T_{\text {noise }}^{\mathrm{h}}+A_{\mathrm{h}} T_{\text {noise }}^{\mathrm{p}}+A_{\mathrm{h}} A_{\mathrm{p}} T_{\text {noise }}^{\mathrm{omt}},
$$

with $A_{\mathrm{p}}$ the mean attenuation of the two polarizer arms. A complete description of derivation and implications of Eq. (1) is given in Carretti et al. (2001). Here we just point out that the offset is generated by OMT and polarizer that partially correlate the antenna noise as well as the sky and atmosphere emissions.

Practically, all the terms are sensitive to thermal fluctuations: $T_{\mathrm{ph}}^{\mathrm{p}}$ by definition; $T_{\text {noise }}^{\mathrm{h}}, T_{\text {noise }}^{\mathrm{p}}$ and $T_{\text {noise }}^{\mathrm{omt}}$ according to the equations (e.g. see Kraus 1986)

$$
\begin{aligned}
& T_{\text {noise }}^{\mathrm{h}}=\left(A_{\mathrm{h}}-1\right) T_{\mathrm{ph}}^{\mathrm{h}} \\
& T_{\text {noise }}^{\mathrm{p}}=\left(A_{\mathrm{p}}-1\right) T_{\mathrm{ph}}^{\mathrm{p}} \\
& T_{\text {noise }}^{\text {omt }}=\left(A_{\mathrm{omt}}-1\right) T_{\mathrm{ph}}^{\mathrm{omt}}
\end{aligned}
$$

with $T_{\mathrm{ph}}^{\mathrm{h}}, T_{\mathrm{ph}}^{\mathrm{p}}, T_{\mathrm{ph}}^{\mathrm{omt}}$ the physical temperatures of horn, polarizer and OMT. Finally, also attenuations $A$ and coefficients $S P_{\text {pol }}$ and $S P_{\text {omt }}$ are functions of the temperature through the relations (see Appendix A for a detailed derivation)

$$
\begin{aligned}
& \Delta A=\frac{A-1}{2 T} \Delta T \\
& \Delta(A-1)=\frac{A-1}{2 T} \Delta T \\
& \Delta\left(S P_{\mathrm{pol}}\right)=\frac{S P_{\mathrm{pol}}}{2 T_{\mathrm{ph}}^{\mathrm{p}}} \Delta T_{\mathrm{ph}}^{\mathrm{p}} \\
& \Delta\left(S P_{\text {omt }}\right)=\frac{A_{\text {omt }}-1}{4 T_{\mathrm{ph}}^{\mathrm{omt}}} S P_{\text {omt }} \Delta T_{\mathrm{ph}}^{\mathrm{omt}} .
\end{aligned}
$$

\footnotetext{
$2 j$ denotes the complex unit.
}

Using Eqs. (1)-(8) we can write the offset variations as a function of those of the physical temperatures as

$$
\begin{aligned}
\Delta(Q+j U)= & H_{\mathrm{h}} \Delta T_{\mathrm{ph}}^{\mathrm{h}} \\
& +H_{\mathrm{p}} \Delta T_{\mathrm{ph}}^{\mathrm{p}} \\
& +H_{\mathrm{omt}} \Delta T_{\mathrm{ph}}^{\mathrm{omt}}
\end{aligned}
$$

where the transfer functions $H$ of the three devices are defined by

$$
\begin{aligned}
& H_{\mathrm{h}}=\frac{3}{2}\left(A_{\mathrm{h}}-1\right)\left[S P_{\mathrm{omt}}\left(1+h^{\mathrm{omt}}\right)+S P_{\mathrm{pol}}\left(1+h^{\mathrm{p}}\right)\right], \\
& H_{\mathrm{p}}=\frac{3}{2} A_{\mathrm{h}}\left[S P_{\mathrm{omt}}\left(A_{\mathrm{p}}-1\right)\left(1+p^{\mathrm{omt}}\right)-S P_{\mathrm{pol}}\left(1+p^{\mathrm{p}}\right)\right], \\
& H_{\mathrm{omt}}=\frac{3}{2} A_{\mathrm{h}} A_{\mathrm{p}}\left(A_{\mathrm{omt}}-1\right) S P_{\mathrm{omt}}\left(1+O^{\mathrm{omt}}\right),
\end{aligned}
$$

with the corrective terms $h, p, O$ given by

$$
\begin{aligned}
& h^{\mathrm{omt}}=\left(A_{\mathrm{p}}-1\right) \frac{T_{\mathrm{ph}}^{\mathrm{p}}}{3 T_{\mathrm{ph}}^{\mathrm{h}}}+A_{\mathrm{p}}\left(A_{\mathrm{omt}}-1\right) \frac{T_{\mathrm{ph}}^{\mathrm{omt}}}{3 T_{\mathrm{ph}}^{\mathrm{h}}}, \\
& h^{\mathrm{p}}=-\frac{T_{\mathrm{ph}}^{\mathrm{p}}}{3 T_{\mathrm{ph}}^{\mathrm{h}}},
\end{aligned}
$$

$$
p^{\mathrm{omt}}=\left(A_{\mathrm{omt}}-1\right) \frac{T_{\mathrm{ph}}^{\mathrm{omt}}}{3 T_{\mathrm{ph}}^{\mathrm{p}}},
$$

$$
\begin{aligned}
p^{\mathrm{p}}= & -\left(\frac{A_{\mathrm{h}}-1}{A_{\mathrm{h}}} \frac{T_{\mathrm{ph}}^{\mathrm{h}}}{3 T_{\mathrm{ph}}^{\mathrm{p}}}+\frac{1}{A_{\mathrm{h}}} \frac{T_{\mathrm{sky}}+T_{\mathrm{atm}}}{3 T_{\mathrm{ph}}^{\mathrm{p}}}\right) \\
O^{\mathrm{omt}}= & \frac{A_{\mathrm{h}}-1}{A_{\mathrm{h}} A_{\mathrm{p}}} \frac{T_{\mathrm{ph}}^{\mathrm{h}}}{6 T_{\mathrm{ph}}^{\mathrm{omt}}}+\frac{A_{\mathrm{p}}-1}{A_{\mathrm{p}}} \frac{T_{\mathrm{ph}}^{\mathrm{p}}}{6 T_{\mathrm{ph}}^{\mathrm{omt}}}+\frac{A_{\mathrm{omt}}-1}{6} \\
& +\frac{1}{A_{\mathrm{h}} A_{\mathrm{p}}} \frac{T_{\mathrm{sky}}+T_{\mathrm{atm}}}{6 T_{\mathrm{ph}}^{\mathrm{omt}}}
\end{aligned}
$$

These corrective terms, dominated by $(A-1)$ terms, are in general much lower than 1 . Slightly different is $h^{\mathrm{p}}$, which can be as high as $\left|h^{\mathrm{p}}\right|=1 / 3$ when $T_{\mathrm{ph}}^{\mathrm{h}}=T_{\mathrm{ph}}^{\mathrm{p}}$, but which, anyway, reduces the instability effects.

The dumping factors with respect to the thermal fluctuations are thus given by noise generation terms $(A-1)$ and by the extra-terms $\left(S P_{\mathrm{omt}}, S P_{\mathrm{pol}}\right)$ typical of the correlation architecture. Actually, the offsets for total power outputs are directly given by $T_{\text {noise }}^{\text {Ant }}$ (Eq. (4)) and the corresponding transfer functions are

$$
\begin{aligned}
H_{\mathrm{h}}^{T P} & =\frac{3}{2}\left(A_{\mathrm{h}}-1\right)\left(1+h^{\mathrm{omt}}\right), \\
H_{\mathrm{p}}^{T P} & =\frac{3}{2} A_{\mathrm{h}}\left(A_{\mathrm{p}}-1\right)\left(1+p^{\mathrm{omt}}\right), \\
H_{\mathrm{omt}}^{T P} & =\frac{3}{2} A_{\mathrm{h}} A_{\mathrm{p}}\left(A_{\mathrm{omt}}-1\right),
\end{aligned}
$$


where the extra terms are not present. Considering that $S P_{\text {omt }}$ and $S P_{\text {pol }}$ can be as low as $10^{-3}$ (see Carretti et al. 2001 for an evaluation of the SPOrt case) the advantages of correlation architectures become obvious.

\section{Effects of fluctuation statistics and SPOrt scanning strategy}

The transfer functions provide the instantaneous response to thermal fluctuations. The relevant effects, however, have to be evaluated on the final maps, which means also the scanning strategy and the behaviour of the thermal fluctuations must be accounted for.

First, we will estimate the dumping factors by an approximate analytical analysis. It is a worst case analysis which does not provide a complete description of the effects - as how the contamination is spread on the various angular scales - but it will help us have an insight into the mechanisms dumping the thermal contaminations.

Then, we will face the exact treatment by simulations which will provide us with the real contamination maps and allow us to estimate the contamination on the signal power spectra.

In this section, we will consider unit offset fluctuations only (arbitrary units), to better evaluate the pure effects irrespective of the real offsets generated by the SPOrt receivers.

\subsection{Contamination in one precession period}

The scanning strategy of SPOrt consists in observing toward the Zenith of the International Space Station while orbiting in $t_{\text {orbit }}=90 \mathrm{~min}$ around the Earth along a $51.6^{\circ}$ inclination orbit. The latter is a circle precessing in $t_{\mathrm{pr}}=70$ days, so that the observation of the sky within declinations $|\delta|<51.6^{\circ}$ is performed in the same time (see Cortiglioni et al. 2004 for details).

The precession moves the trajectory by the $7^{\circ}$ of the beamsize along the Celestial Equator in $N_{\text {orbit }} \sim 22$ orbits, during which SPOrt observes the same pixel stripe.

The map-making procedure consists in averaging all the data collected in a pixel (see Sbarra et al. 2003 for details). Thus the error $\Delta(Q+j U)_{\text {scan }}$ on the maps after $N_{\text {orbit }}$ observations is given by

$\Delta(Q+j U)_{\mathrm{scan}}=\frac{1}{N_{\text {orbit }}} \sum_{i} \Delta(Q+j U)\left(t_{i}\right)$,

where $t_{i}$ is the time at the $i$ th of the $N_{\text {orbit }}$ passages.

The result depends on the behaviour of the fluctuations. With no loss of generality, we can consider sinusoidal variations for the temperature of each device

$\Delta T=\Delta T_{0} \cos \left(2 \pi f_{\mathrm{tf}} t\right)$,

since a generic function can always be expanded by Fourier transform ( $f_{\mathrm{tf}}$ is the fluctuation frequency). The linear relationship between temperature and offset variations (Eq. (9)) leads to the same behaviour of the offset fluctuations

$\Delta(Q+j U)=\Delta(Q+j U)_{0} \cos \left(2 \pi f_{\mathrm{tf}} t\right)$.
From a statistical point of view, fluctuations can be divided in three different types:

1. Thermal fluctuations synchronous with the Sun illumination (i.e. the ISS day). In this case the fluctuation frequency $f_{\mathrm{tf}}$ is that of the ISS day

$$
\begin{aligned}
f_{\text {tf }} & =f_{\text {day }} \\
& =f_{\text {orbit }}+f_{\text {pr }}-f_{\odot},
\end{aligned}
$$

i.e. the orbit frequency $f_{\text {orbit }}=1 / t_{\text {orbit }}$ properly corrected for the ISS precession $\left(f_{\mathrm{pr}}\right)$ and the Sun revolution $\left(f_{\odot}\right)$. This is the natural behaviour when no active temperature control is in operation, with the maximum expected under Sun illumination and the minimum when in the Earth shadow. In this case no dumping over the $N_{\text {orbit }}$ orbits occurs since, when the instrument is observing toward the same sky pixel during these consecutive orbits, the fluctuation has the same phase and the averaging does not dump the effects on the pixel map (see Mennella et al. 2002). In this case dumping is simply by a unit factor

$D_{\text {scan }}^{1}=1$.

2. Thermal fluctuations synchronous with the Sun illumination, but with a frequency which is an integer multiple of the ISS day

$f_{\text {tf }}=K f_{\text {day }}, \quad$ with $K$ integer.

This situation can occur when an active control is in operation, but without enough strength to decorrelate the period with respect to the Sun illumination. In this case again no dumping occurs over the $N_{\text {orbit }}$ orbits, and the corresponding dumping factor is

$D_{\text {scan }}^{K}=1$.

3. Thermal fluctuations asynchronous with the night-day cycle

$f_{\text {tf }} \neq K f_{\text {day }}$.

This situation is typical of cryogenic sections with closed loop control, where the stabilized temperature has a behaviour imposed by the control electronics and in general is independent of the outer temperature fluctuations. In this case the situation is better: as described by Mennella et al. (2002) there is a dumping by a factor

$D_{\text {scan }}^{\text {asy }}=1 / N_{\text {orbit }}$,

which greatly reduces the effects on the final maps. We would like to add only that, differing from the others, the value of this dumping factor is a conservative estimate, since it represents the envelope of actual $K$ values (see Mennella et al. 2002 for a detailed discussion).

Table 1 reports the worst case fluctuations on the final maps after a precession time (in arbitrary units relative to the amplitude in a single orbit)

$\Delta(Q+j U)_{\text {scan }}=D_{\text {scan }}^{x} \Delta(Q+j U)$ 
Table 1. Fluctuations on the map after 1 precession time for $7^{\circ}$ pixels. Values for both synchronous and asynchronous thermal instabilities are reported (see text for details).

\begin{tabular}{lccc}
\hline \hline$f_{\text {tf }}$ & $N_{\text {orbit }}$ & $\begin{array}{c}\Delta(Q+j U)_{0} \\
{[1 \text { orbit }]}\end{array}$ & $\begin{array}{c}\Delta(Q+j U)_{\text {scan }} \\
{\left[N_{\text {orbit }} \text { orbits }\right]}\end{array}$ \\
\hline$f_{\text {tf }}=f_{\text {day }}$ & 22 & 1 & 1 \\
$f_{\text {tf }}=K f_{\text {day }}$ & 22 & 1 & 1 \\
$f_{\text {tf }} \neq K f_{\text {day }}$ & 22 & 1 & 0.046 \\
\hline
\end{tabular}

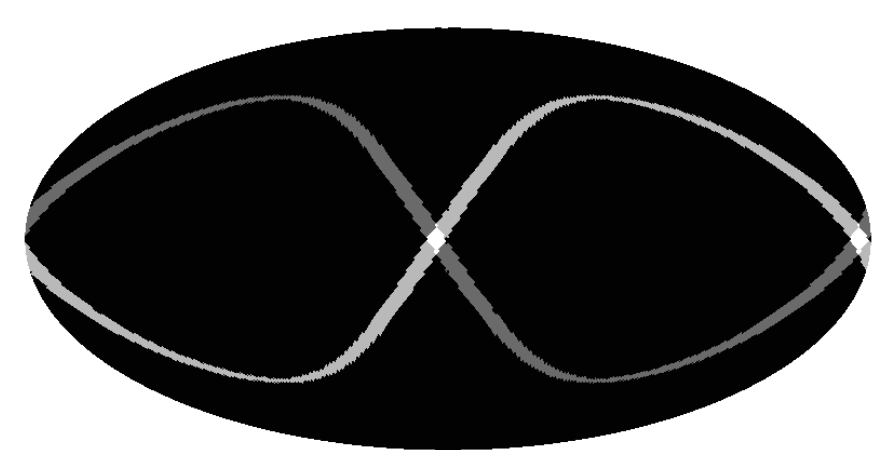

Fig. 1. Sky coverage of two orbits separated by 35 days (dark and light gray). The $180^{\circ}$-shift is due to the precession. The central pixel is observed during both orbits. The map is a mollview projection in Celestial coordinates.

with $x=K$ or $x=$ asy, and shows the advantage to be asynchronous with the Sun illumination.

Actually, in one precession each pixel is visited twice: once during the first 35 days and a second time after about half a precession (see Fig. 1). Since the combination of these two sets of observations does not play a relevant role in our worst case analysis, we will discuss it at the end of Sect. 3.2.

\subsection{Contamination in the lifetime}

So far we have analyzed dumping after just one sky coverage. As described above, SPOrt observes the whole of the accessible sky every 70 days and the final map comes from the combination of these coverages. The thermal effects on the final map thus depend on how the contamination maps of each coverage combine with each other.

In the simple case $f_{\text {tf }}=f_{\text {day }}$ the fluctuations depend on the Sun illumination and show the maximum temperature when in presence of the Sun and the minimum when in the Earth shadow. Over a precession period, SPOrt observes each pixel for $N_{\text {orbit }}$ consecutive orbits. 70 Earth-days later, when the precession allows SPOrt to observe the same pixel again, the illumination conditions are different due to the new position of the Sun with respect to the Earth. Thus, each pixel will be observed when temperature fluctuations are in different phases depending on the observing season, which provides some cancellation effects. As an example, if a pixel is observed during the day (Sun above the horizon) with a positive temperature fluctuation, 6 months later it is observed at night with a negative temperature variation.
Table 2. Dumping factors due to the precession of the SPOrt orbit. The cases of thermal behaviour synchronous with the Sun illumination with $K=1\left(D_{\mathrm{pr}}^{1}\right), K=10\left(D_{\mathrm{pr}}^{10}\right)$ and $K=30\left(D_{\mathrm{pr}}^{30}\right)$ are displayed. The asynchronous case $\left(D_{\mathrm{pr}}^{\mathrm{asy}}\right)$ is shown as well.

\begin{tabular}{cccccc}
\hline \hline $\begin{array}{l}\text { Lifetime } \\
\text { [months] }\end{array}$ & $N_{\mathrm{pr}}$ & $D_{\mathrm{pr}}^{1}$ & $D_{\mathrm{pr}}^{10}$ & $D_{\mathrm{pr}}^{30}$ & $D_{\mathrm{pr}}^{\text {asy }}$ \\
\hline 12 & 5 & 0.046 & 0.745 & 0.201 & 0.200 \\
18 & 8 & 0.220 & 0.416 & 0.002 & 0.125 \\
24 & 10 & 0.046 & 0.191 & 0.141 & 0.100 \\
30 & 13 & 0.135 & 0.079 & 0.078 & 0.077 \\
36 & 15 & 0.045 & 0.183 & 0.065 & 0.067 \\
\hline
\end{tabular}

In fact, as in Eq. (15), the error on the final map is the average of the offset variations in each observation time:

$\Delta(Q+j U)_{\mathrm{fm}}=\frac{1}{N_{\mathrm{pr}}} \sum_{i} \Delta(Q+j U)\left(t_{i}\right)$,

where $N_{\mathrm{pr}}$ is the number of precessions and $t_{j}$ the observing time. Two different observations of the same pixel differ for a multiple of both the orbit time $t_{\text {orbit }}$ and the precession time $t_{\mathrm{pr}}$. Thus, the $i$ th observation of a pixel happens at the time

$$
\begin{aligned}
t_{i} & =t_{\mathrm{px}}+\Delta t_{i} \\
& =t_{\mathrm{px}}+n_{i} t_{\mathrm{orbit}} \\
& =t_{\mathrm{px}}+i t_{\mathrm{pr}},
\end{aligned}
$$

where $i$ is the $i$ th precession, $n_{i}$ the number of orbits at this precession and $t_{\mathrm{px}}$ the time when the first observation of the pixel occurred. Using these expressions for valid $t_{i}$, Eq. (25) becomes

$$
\begin{aligned}
& \Delta(Q+j U)_{\mathrm{fm}}=D_{\mathrm{pr}}^{1} \Delta(Q+j U)_{0} \\
& D_{\mathrm{pr}}^{1}=\frac{\cos \alpha}{N_{\mathrm{pr}}} \sum_{i=0}^{N_{\mathrm{pr}}-1} \cos \beta_{i}+\frac{\sin \alpha}{N_{\mathrm{pr}}} \sum_{i=0}^{N_{\mathrm{pr}}-1} \sin \beta_{i} \\
& \alpha=2 \pi f_{\mathrm{day}} t_{\mathrm{px}} \\
& \beta_{i}=2 \pi i \frac{f_{\odot}}{f_{\mathrm{pr}}} .
\end{aligned}
$$

$D_{\mathrm{pr}}^{1}$ is the dumping factor related to the precession with respect to the instantaneous offset amplitude $\Delta(Q+j U)_{0}$. It depends not only on the orbital parameters, but also on the pixel position (by $t_{\mathrm{px}}$ ). In order to evaluate the effects we consider the worst case, i.e. the maximum value of $D_{\mathrm{pr}}^{1}$. Table 2 reports its value for different mission lifetimes.

It is worth noting that the best situation corresponds to 12 , 24 and 36 months, during which the mission benefits from complete Sun revolutions. To understand this, one has to bear in mind that the phase of the fluctuation at a given pixel depends on the season and performs a whole $2 \pi$ cycle in one year. When the lifetime is close to a complete Sun revolution $(12,24$, 36 months), the phases taken at the various precessions sample well the whole cycle and the net effect is a cancellation of the thermal contributions. On the other hand, when the lifetime is 18 or 30 months, the last half of a year covers just a half of the whole cycle and the cancellation is not optimal. 
Moving to the second kind of fluctuations $-f_{\text {tf }}=K f_{\text {day }}-$ similar considerations can be done and the errors on the final maps $\Delta(Q+j U)_{\mathrm{fm}}^{K}$ are given by:

$$
\begin{aligned}
\Delta(Q+j U)_{\mathrm{fm}}^{K}=D_{\mathrm{pr}}^{K} \Delta(Q+j U)_{0} & \\
D_{\mathrm{pr}}^{K}= & \frac{\cos \alpha^{K}}{N_{\mathrm{pr}}} \sum_{i=0}^{N_{\mathrm{pr}}-1} \cos \beta_{i}^{K} \\
& +\frac{\sin \alpha^{K}}{N_{\mathrm{pr}}} \sum_{i=0}^{N_{\mathrm{pr}}-1} \sin \beta_{i}^{K} \\
\alpha^{K}= & 2 \pi K f_{\mathrm{day}} t_{\mathrm{px}} \\
\beta_{i}^{K}= & 2 \pi K i \frac{f_{\odot}}{f_{\mathrm{pr}}} .
\end{aligned}
$$

The worst case is again the maximum value of the dumping factor $D_{\mathrm{pr}}^{K}$ and is reported in Table 2 for different lifetimes and a couple of $K$ values (namely, $K=10,30$ ). While for $K=1$ the phase does a complete $2 \pi$ period in one Sun revolution, for $K>1$ the cycle is closed in $1 / K$ years and, for each pixel, the phase sampling may not be complete and well distributed over the whole period. Thus, the $K>1$ case is less predictable and in some pixels (Table 2 is only the worst case in the whole map) the fluctuations may be only slightly dumped, as occurs for $K=10$ and lifetime $=12$ months. On the other hand, in a few cases (as for $K=30$, lifetime $=18$ months) even the worst pixel shows a large dumping. Anyway, in general, the maximum fluctuations are worse than in the simplest $K=1$ case.

Finally, in the case of asynchronous fluctuations the same situation as the $N_{\text {orbit }}$ consecutive asynchronous scans occurs, resulting in a dumping factor by the number of precessions $N_{\mathrm{pr}}$

$D_{\mathrm{pr}}^{\text {asy }}=1 / N_{\mathrm{pr}}$.

Results for various lifetimes are again reported in Table 2.

The total reduction on the final map includes the actions of both the first $N_{\text {orbit }}$ scans and the precession, and is given by the product

$D^{x}=D_{\mathrm{scan}}^{x} D_{\mathrm{pr}}^{x}$,

with $x=K$ or $x=$ asy. It represents the total efficiency with which the scanning strategy is able to dump the signal fluctuations generated by the system. The corresponding offset fluctuations

$\Delta(Q+j U)_{\mathrm{fm}}=D^{x} \Delta(Q+j U)_{0}$

are reported in Table 3 for two different lifetimes.

However, these values represent the residual pixel fluctuation only for the worst pixel. Accounting for neither the statistical nor angular distribution of the deviations, they do not provide a complete description of the impact of thermal fluctuations.

As a final consideration, we recall that in one precession the pixels are in general visited twice: once during the first 35 days and a second time after about half a precession (the exact delay depends on the distance of the pixel from the Equator). These two sets of observations can be treated as observations of two different pixels and combined only at the end to evaluate the
Table 3. Fluctuations on the final map due to both the first $N_{\text {orbit }}$ scans and the precession. The values are reported for the four cases described in Table 2 treated in the text and for two different lifetimes. All the fluctuations are in arbitrary units considering $\left|\Delta(Q+j U)_{0}\right|=1$ the maximum offset fluctuation in one orbit.

\begin{tabular}{lcc}
\hline \hline$f_{\text {tf }}$ & $\begin{array}{c}\Delta(Q+j U)_{\mathrm{fm}} \\
{[12 \text { months }]}\end{array}$ & $\begin{array}{c}\Delta(Q+j U)_{\mathrm{fm}} \\
{[36 \text { months }]}\end{array}$ \\
\hline$f_{\text {day }}$ & 0.046 & 0.045 \\
$10 f_{\text {day }}$ & 0.745 & 0.183 \\
$30 f_{\text {day }}$ & 0.201 & 0.065 \\
$f_{\text {tf }} \neq K f_{\text {day }}$ & 0.009 & 0.003 \\
\hline
\end{tabular}

total dumping factor. They are characterized by two different parallactic angles and their combination can lead to some cancellation. In fact, when the difference between the parallactic angles is $0^{\circ}$ no cancellation occurs; when the difference is $90^{\circ}$, the offset is cancelled out. For the SPOrt scanning strategy, this difference runs between $0^{\circ}$ (at the top and bottom edges of the orbit) and $103.2^{\circ}$, i.e. twice the orbit inclination (at the Equator). Thus, in our worst case analysis no further cancellation has to be considered.

Anyway, a complete treatment of the SPOrt case must be done via simulations, that, reproducing the real scanning strategy and map-making, account for all the details.

\subsection{Contamination on angular power spectra}

A complete description of thermal fluctuation effects is provided by the angular power spectra of the contamination maps. To estimate these power spectra we simulate the experiment assuming the offset fluctuations of Eq. (17) and generating the final $Q, U$ maps using the SPOrt map-making procedure (Sbarra et al. 2003), which also accounts for pixel observations at different parallactic angles. We adopt a unit fluctuation amplitude $\left|\Delta(Q+j U)_{0}\right|=1$ (arbitrary units) to evaluate relative effects.

Figure 2 reports the results for a 12-month lifetime. Clearly, the best case is the asynchronous one where the fluctuations are well dumped on all the scales of interest for SPOrt $(\ell<$ $25)$ reaching values $3.5-5$ orders of magnitude lower than the instantaneous fluctuation amplitude $\left(\sqrt{\ell(\ell+1) C_{\ell} /(2 \pi)}\right.$ is a fair estimate of the signal).

The synchronous case with $K=1$ reduces the contamination as well, but the dumping is limited to about 2.5 orders of magnitude.

The synchronous case with $K>1$ is interesting: dumping is relevant up to a critical $\ell_{K}$ at which the fluctuations dramatically increase at a level much higher than those for $K=1$. This confirms the results of the analytical analysis (see Table 2), where we find that cases with $K>1$ can show maximum deviations larger than for $K=1$. But much more interesting is the relation between the critical $\ell_{K}$ and the fluctuation frequency: the higher $K$, the higher $\ell_{K}$. That is, the high frequency of the thermal fluctuations brings the power of the contamination to small angular scales: approximately, a $K=1$ behaviour generates dipole-like patterns, while, for a generic $K$, the dominant structures are approximately on $\theta=180^{\circ} / K$ scale. This is 

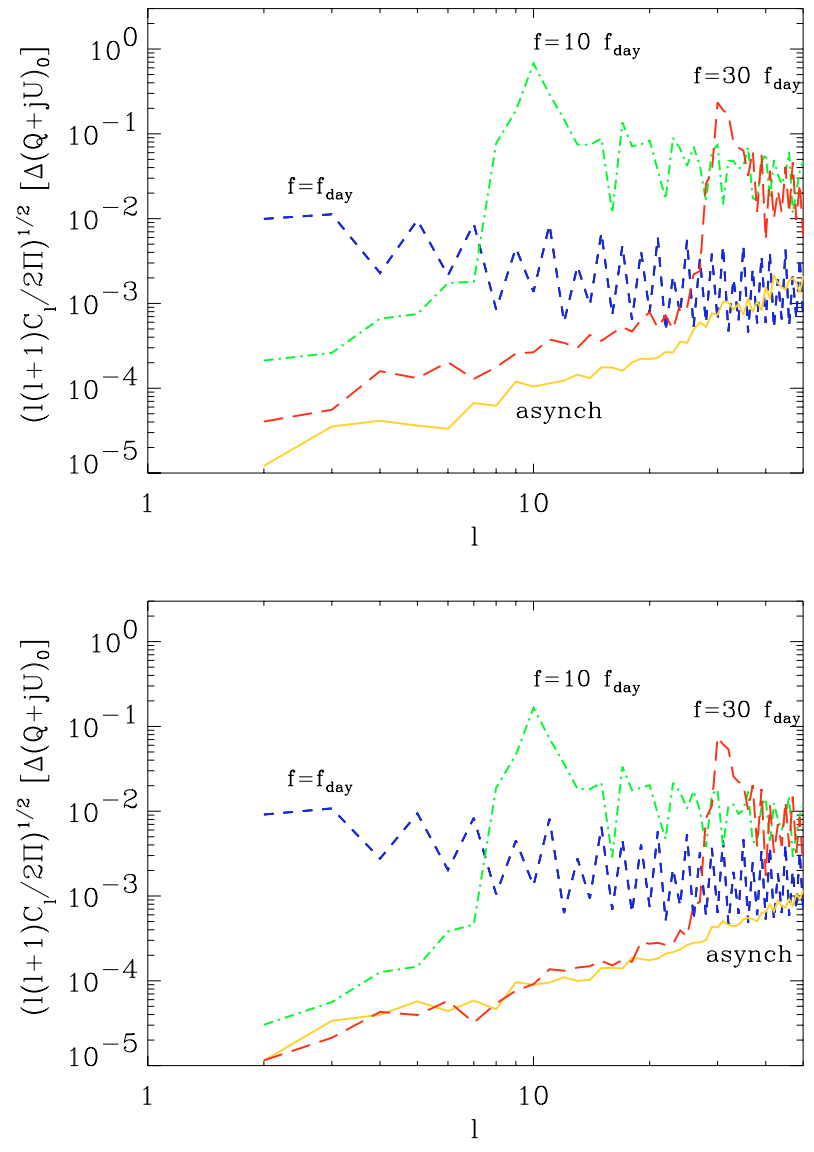

Fig. 2. Top: $E$-mode power spectra of the contamination of the thermal fluctuations featuring a unit amplitude $\left|\Delta(Q+j U)_{0}\right|=1$. A lifetime of 12 months is assumed. The spectra are presented for four different configurations: synchronous with the Sun illumination ( $f_{\text {tf }}=K f_{\text {day }}$ with $K=1,10,30$ ), and asynchronous. For synchronous cases we plot the average of realizations with different polarization angles of $\Delta(Q+j U)_{0}$. The asynchronous case, instead, is the average of many realizations with $K$ in the $[30.2,30.8]$ range centred on 30.5. Ranges with other central frequencies provide similar results. Bottom: the same but for a 36-month lifetime.

confirmed by the spectral shapes of Fig. 2 where the peak of the spectra are at $\ell_{10} \sim 10$ for $K=10$ and at $\ell_{30} \sim 30$ for $K=30$.

The cleanliness of the $K=30$ configuration for $\ell<25$ is appealing: all the $\ell$-range accessible to SPOrt has low contamination (about 1-2 orders of magnitude lower than the case with $K=1$ ), making the condition $K>30$ an interesting option for the experiment.

The maps in Fig. 3, reporting the polarized intensity $I_{\mathrm{p}}=$ $\sqrt{Q^{2}+U^{2}}$ of the contamination with 12-month lifetime, support this view giving an insight from the pixel-space point of view. The patterns look different depending on the $K$ value and show that the higher $K$, the smaller the size of the dominant structures. Thus, a $K$ value large enough to make the dominant structures on scales smaller than $7^{\circ}$ (SPOrt's $F W H M$ ) allows the minimization of the thermal fluctuation impact in the angular-scale range of interest for SPOrt.
Table 4. Temperature and fluctuations (maximum amplitude) of the SPOrt antenna devices.

\begin{tabular}{lcc}
\hline \hline Device & $T_{\mathrm{ph}}[\mathrm{K}]$ & $\Delta T_{\mathrm{ph}}[\mathrm{K}]$ \\
\hline Horn & 300 & $<0.2$ \\
Polarizer & 80 & $<0.1$ \\
OMT & 80 & $<0.1$ \\
\hline
\end{tabular}

It is worth noting that the maximum values of the fluctuations in Fig. 3 are close to those of Table 2, in agreement with the analytical analysis.

The results for a longer lifetime (36 months) are shown in Fig. 2 as well, and their comparison with 12-month spectra gives us a hint to the time behaviour. The case $K=1$ is practically unchanged, confirming that a longer experiment does not provide a benefit in case of synchronous fluctuations with $K=1$ (see Table 2).

The cases $K>1$, instead, show a decrement of the thermal fluctuation contamination, confirming that this configuration is good for the SPOrt case, at least for $K>30$. Finally, the asynchronous case does not show any improvement, in contrast with the prediction of Table 2 . The numerical error limit is likely to have already been reached.

\section{E-mode contamination in the SPOrt case}

The SPOrt experiment has two well defined thermal environments (see also Table 4):

- the horn location with temperature $T_{\mathrm{ph}}^{\mathrm{h}} \sim 300 \mathrm{~K}$. Simulations considering the ISS environment show that the natural thermal fluctuations, with no thermal control, are synchronous with the Sun illumination $\left(f_{\mathrm{tf}}=f_{\text {day }}\right)$ with amplitude $\Delta T \sim 3 \mathrm{~K}$. However, the adoption of an active control performed through heaters ensures a stability within $\Delta T_{\mathrm{ph}}^{\mathrm{h}}<0.2 \mathrm{~K}$ and breaks the correlation with the Sun modulation, moving the fluctuations to shorter periods with frequencies $f_{\mathrm{tf}}>30 f_{\text {day }}$ for all the SPOrt radiometers. This occurs already for the $22 \mathrm{GHz}$, which, having the larger heat capacity, is the slowest. In addition, the fluctuations are decoupled from the Sun illumination modulation showing an asynchronous behaviour.

- the cryogenic part, including polarizer and OMT, with temperature $T_{\mathrm{ph}}^{\text {cryo }} \sim 80 \mathrm{~K}$ and thermal stability within $\Delta T_{\mathrm{ph}}^{\text {cryo }}<0.1 \mathrm{~K}$ provided by a closed-loop cryo-cooler. These fluctuations are intrinsically uncorrelated with the Sun illumination.

The presence of two thermal environments suggests to rewrite Eq. (9) as

$$
\Delta(Q+j U)=H_{\mathrm{h}} \Delta T_{\mathrm{ph}}^{\mathrm{h}}+\left(H_{\mathrm{p}}+H_{\mathrm{omt}}\right) \Delta T_{\mathrm{ph}}^{\mathrm{cryo}}
$$

and to discuss their effects separately. 
$\mathrm{K}=1$

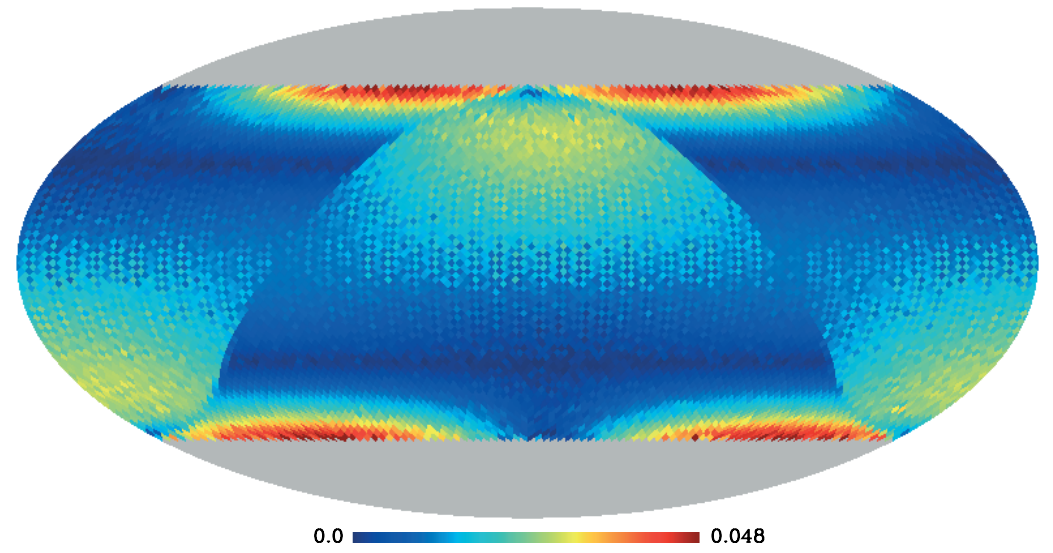

$\mathrm{K}=10$

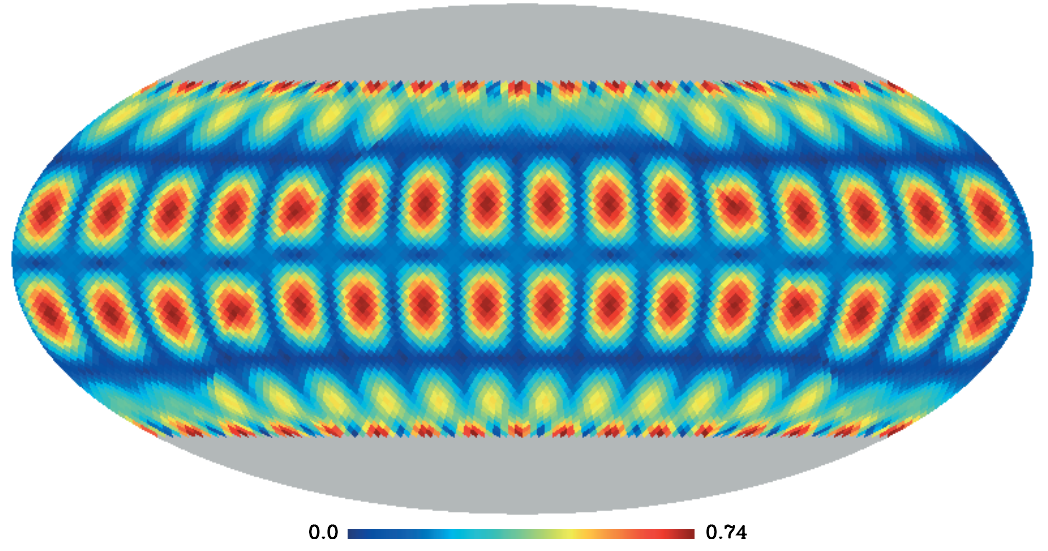

$\mathrm{K}=30$

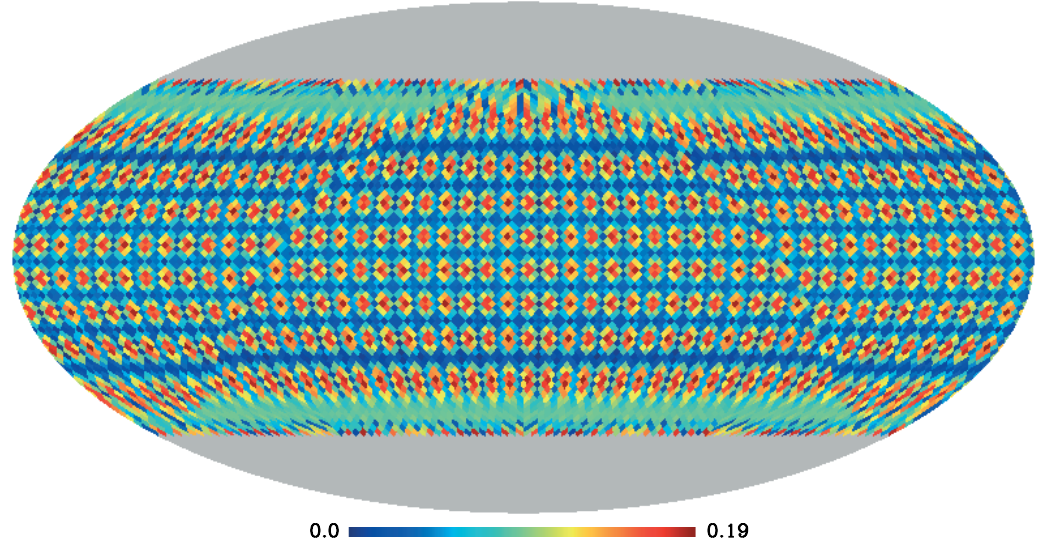

Fig. 3. Polarized intensity $I_{\mathrm{p}}=\sqrt{Q^{2}+U^{2}}$ map of the thermal contamination for a 12-month lifetime. The cases $K=1$ (top), $K=10$ (mid) and $K=30$ (bottom) are presented. All fluctuations are in arbitrary units considering $\left|\Delta(Q+j U)_{0}\right|=1$ the amplitude in one orbit.

\subsection{Temperature fluctuations of the horn}

The offset fluctuations induced by the horn alone are given by

$$
\begin{aligned}
\Delta(Q+j U)= & \frac{3}{2}\left(A_{\mathrm{h}}-1\right) \\
& \times\left[S P_{\mathrm{omt}}\left(1+h^{\mathrm{omt}}\right)+S P_{\mathrm{pol}}\left(1+h^{\mathrm{p}}\right)\right] \Delta T_{\mathrm{ph}}^{\mathrm{h}} .
\end{aligned}
$$

Table 5 lists the values computed for the SPOrt receivers at 22 and $90 \mathrm{GHz}$, representing the best and worst cases, respectively.

A low sensitivity is clearly shown by the transfer functions, whose levels reduce the impact of thermal instabilities by $4-5$ orders of magnitude. The offset fluctuations are directly related to the values of the OMT isolation and the differential attenuation between the two main polarizations of the polarizer 
Table 5. Maximum offset fluctuation $\Delta(Q+j U)$ in one orbit $(90 \mathrm{~min})$ induced by the horn temperature instabilities for the SPOrt experiment. The cases of the 22 and $90 \mathrm{GHz}$ channels are reported, representing the best and worst cases among the SPOrt receivers. Details about horn temperature fluctuations $\left(\Delta T_{\mathrm{ph}}^{\mathrm{h}}\right)$ and antenna characteristics are also listed: horn attenuation $\left(A_{\mathrm{h}}\right)$, OMT isolation $\left(\left|S_{B 1}\right|^{2}\right)$, polarizer differential attenuation $\left(A_{\perp}-A_{\|}\right), S P_{\text {omt }}$ and $S P_{\text {pol }}$ coefficients, and the $H_{\mathrm{h}}$ transfer function. $S P_{\text {omt }}$ has been estimated with the approximation $S P_{\text {omt }} \sim$ $2 A_{\text {omt }}\left|S_{A 1} S_{B 1}^{*}\right|$ as described in Carretti et al. (2001). The case of a $3 \mathrm{~K}$ variation is also shown, that is the natural horn temperature fluctuation without active control.

\begin{tabular}{lccccccccc}
\hline \hline Configuration & $\begin{array}{c}v \\
{[\mathrm{GHz}]}\end{array}$ & $\begin{array}{c}\Delta T_{\mathrm{ph}}^{\mathrm{h}} \\
{[\mathrm{K}]}\end{array}$ & $\begin{array}{c}A_{\mathrm{h}} \\
{[\mathrm{dB}]}\end{array}$ & $\begin{array}{c}\left|S_{B 1}\right|^{2} \\
{[\mathrm{~dB}]}\end{array}$ & $\begin{array}{c}A_{\perp}-A_{\|} \\
{[\mathrm{dB}]}\end{array}$ & $S P_{\text {omt }}$ & $S P_{\text {pol }}$ & $H_{\mathrm{h}}$ & $\Delta(Q+j U)$ \\
\hline $22 \mathrm{GHz}$ & 22 & \pm 0.2 & 0.05 & -65 & -33 & $1.15 \times 10^{-3}$ & $2.45 \times 10^{-4}$ & $2.40 \times 10^{-5}$ & 4.8 \\
$90 \mathrm{GHz}$ & 90 & \pm 0.2 & 0.1 & -60 & -30 & $2.05 \times 10^{-3}$ & $4.89 \times 10^{-4}$ & $8.75 \times 10^{-5}$ & 17.5 \\
$22 \mathrm{GHz}(3 \mathrm{~K})$ & 22 & \pm 3 & 0.05 & -65 & -33 & $1.15 \times 10^{-3}$ & $2.45 \times 10^{-4}$ & $2.40 \times 10^{-5}$ & 72.0 \\
$90 \mathrm{GHz}(3 \mathrm{~K})$ & 90 & \pm 3 & 0.1 & -60 & -30 & $2.05 \times 10^{-3}$ & $4.89 \times 10^{-4}$ & $8.75 \times 10^{-5}$ & 262.0 \\
\hline
\end{tabular}

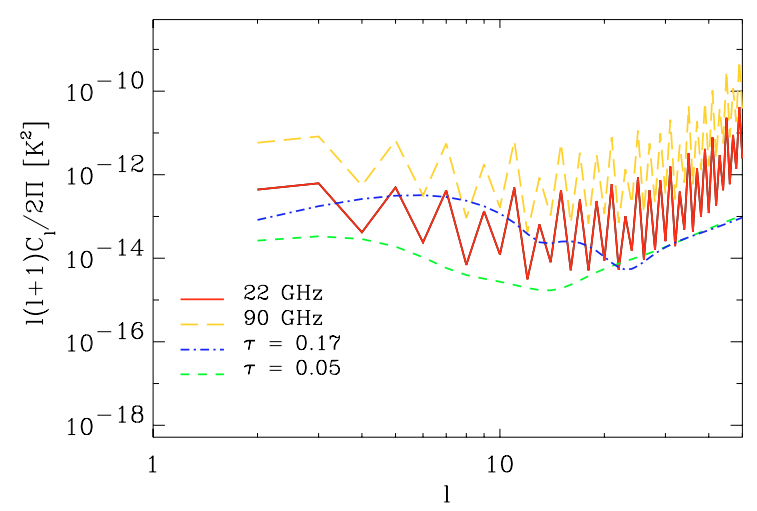

Fig. 4. E-mode power spectra of the contamination induced by thermal fluctuations of the horn in case of no temperature control. Spectra are corrected for the smearing effects of the beam window function. A lifetime of 36 months is considered. The $E$-mode spectrum expected for the cosmological signal is also reported for comparison for two cosmological models: the concordance model as from WMAP's first-year results with $\tau=0.17$ (Spergel et al. 2003); a similar model but with a lower $\tau=0.05$.

(see Eqs. (2) and (3)). Such low offset fluctuations are due to the improvements in performances of passive devices obtained by the SPOrt team (Cortiglioni et al. 2004; Peverini et al. 2003). In fact, state-of-the-art OMTs available at the beginning of the project had isolations worse than $40 \mathrm{~dB}$, while the device developed for this experiment, with about $60 \mathrm{~dB}$ isolation, leads to a decrease of the offset fluctuations by a factor about 10 .

As described in Sect. 3, the evaluation of the thermal instability impact has to be performed in the multipole space, where the contamination on different angular scales can be estimated and compared to the expected cosmological signal.

First of all we consider what happens in the case of no active control, corresponding to a synchronous behaviour with $K=1$. The contamination-map power spectra are shown in Fig. 4, along with the expected polarized sky emission ( $E$-mode) as from WMAP best-fit cosmological model (Spergel et al. 2003; Kogut et al. 2003). The cosmological signal appears significantly contaminated even when the optical depth of the reionized medium is $\tau=0.17$. This means that the free thermal fluctuations induced by the ISS environment are too large for such a measurement, calling for a reduction of the thermal disturbances.
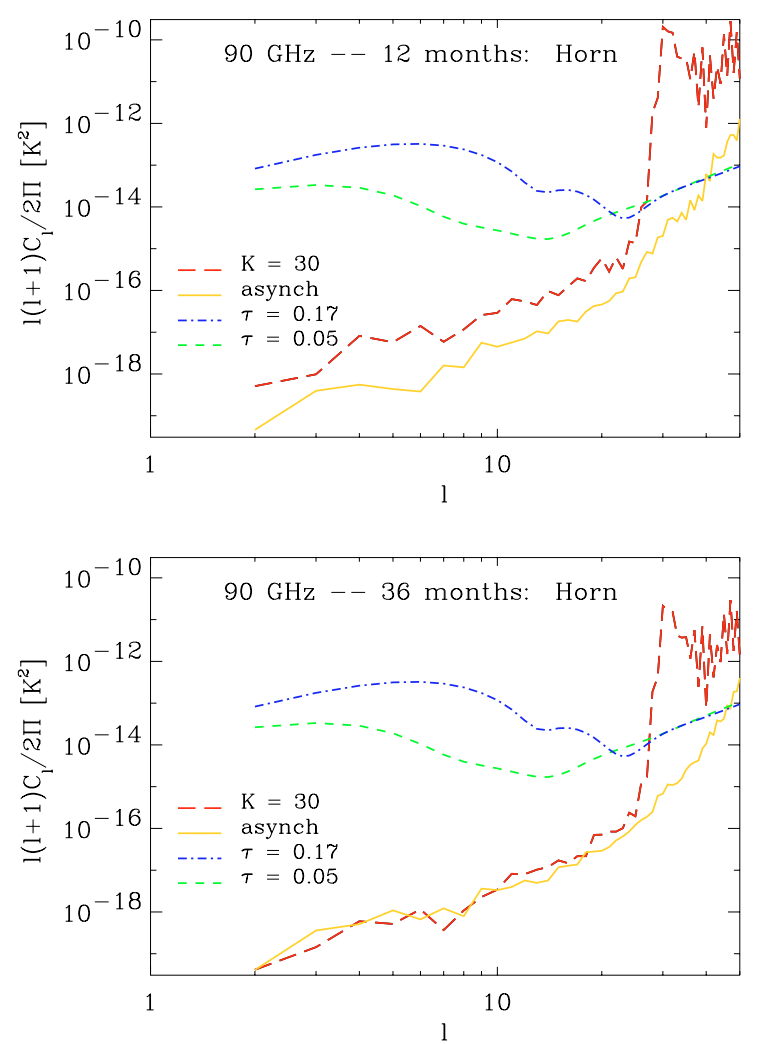

Fig. 5. Top: $E$-mode power spectra of the contamination due to horn thermal fluctuations with active temperature control. Spectra are corrected for the smearing effects of the beam window function. The $90 \mathrm{GHz}$ receiver and a lifetime of 12 months are assumed. The $E$-mode spectrum expected for the cosmological signal is also reported for comparison for two cosmological models: that from WMAP first-year results with $\tau=0.17$ (Spergel et al. 2003); a similar model but with a lower $\tau=0.05$. Bottom: the same for a 36-month lifetime.

The active control adopted for SPOrt allows temperature fluctuations with amplitude $\Delta T_{\mathrm{ph}}^{\mathrm{h}}<0.2 \mathrm{~K}$ and with frequency $f_{\text {tf }}>30 f_{\text {day }}$, which satisfies the condition identified in Sect. 3 . The power spectrum of the contamination for this configuration is reported in Fig. 5, where the worst case $(90 \mathrm{GHz}$ receiver) is shown for two different lifetimes (12 and 36 months). Here we consider both a synchronous behaviour with $K=$ 30 and the case of asynchronous fluctuations. The main result is that already for $K=30$ and a 12-month lifetime the 
Table 6. As for Table 5, but for the cryo-stage of the SPOrt radiometers. As a worst case, we assume $A_{\mathrm{p}}=0.1 \mathrm{~dB}$ and $A_{\text {omt }}=0.2 \mathrm{~dB}$ the attenuations of polarizer and OMT, respectively. Anyway, the offset fluctuations are marginally dependent on their values, $S P_{\mathrm{pol}}$ being the dominant term.

\begin{tabular}{lccccccccc}
\hline \hline Configuration & $\begin{array}{c}\Delta T_{\mathrm{ph}}^{\text {cryo }} \\
{[\mathrm{K}]}\end{array}$ & $\begin{array}{c}A_{\mathrm{h}} \\
{[\mathrm{dB}]}\end{array}$ & $\begin{array}{c}\left|S_{B 1}\right|^{2} \\
{[\mathrm{~dB}]}\end{array}$ & $\begin{array}{c}A_{\perp}-A_{\|} \\
{[\mathrm{dB}]}\end{array}$ & $S P_{\text {omt }}$ & $S P_{\mathrm{pol}}$ & $H_{\mathrm{p}}$ & $H_{\text {omt }}$ & $\Delta(Q+j U)$ \\
& \pm 0.1 & 0.05 & -65 & -33 & $1.15 \times 10^{-3}$ & $2.45 \times 10^{-4}$ & $3.21 \times 10^{-4}$ & $8.62 \times 10^{-5}$ & 40.7 \\
\hline $22 \mathrm{GHz}$ & \pm 0.1 & 0.1 & -60 & -30 & $2.05 \times 10^{-3}$ & $4.89 \times 10^{-4}$ & $6.46 \times 10^{-4}$ & $1.56 \times 10^{-4}$ & 80.2 \\
$90 \mathrm{GHz}$ & & & & & & & & &
\end{tabular}

contamination is well below the signal, not only for $\tau=0.17$, but even for a lower optical depth $\tau=0.05$. A 36-month lifetime provides even better results. However, the 12-month lifetime already provides a very comfortable scenario which does not need any specific data cleaning.

\subsection{Temperature fluctuations of the cryo-stage}

The cryo-stage induces offset fluctuations according to the formula

$\Delta(Q+j U)=\left(H_{\mathrm{p}}+H_{\mathrm{omt}}\right) \Delta T_{\mathrm{ph}}^{\mathrm{cryo}}$.

The values for the 22 and $90 \mathrm{GHz}$ receivers are reported in Table 6 .

In this case the dominant term is related to the polarizer and only $S P_{\text {pol }}$ acts to dump the fluctuation, without contributions from $(A-1)$ terms. As already mentioned by Carretti et al. (2001), the leading offset due to the polarizer is a polarized noise generated by the device itself, rather than the correlation of an unpolarized noise generated by the antenna. This is why only one dumping factor is in action $\left(S P_{\mathrm{pol}}\right)$, leading to the quite unexpected result that the cryo-stage generates the most relevant contamination, larger than the horn contribution, even though the latter is in a warm section.

The behaviour is determined by the cooler electronics, which, performing an active control to anchor the temperature to a fixed set-point, induces fluctuations not related with the external environment. This is true only as a first approximation, since a small correlation can arise due to the coupling between the cryo-stage and the feed horn. In fact, these are connected through a transition waveguide whose aim is to thermally separate the two environments while allowing the passage of electromagnetic waves. However, the thermal separator filters the fluctuations and only a small fraction is transmitted to the cold-stage. As an example, tests performed during the integration phases on the BaR-SPOrt receiver, an instrument similar to SPOrt (Cortiglioni et al. 2003), show that fluctuations of the cold-stage induced by the horn are a few percent of those of the horn itself. Therefore, considering a $0.2 \mathrm{~K}$ horn temperature variation, the fluctuations correlated to the warm part are expected to be no more than few hundredths of Kelvin. Anyway, should a coupling with the horn arise, the fluctuations would have a statistics similar to that of the horn, leading to, as worst case, a periodic behaviour with $f_{\mathrm{tf}}>30 f_{\text {day }}$.

As for the horn section, we study the contamination impact through the analysis of the power spectrum. We perform the analysis for two configurations: the asynchronous behaviour
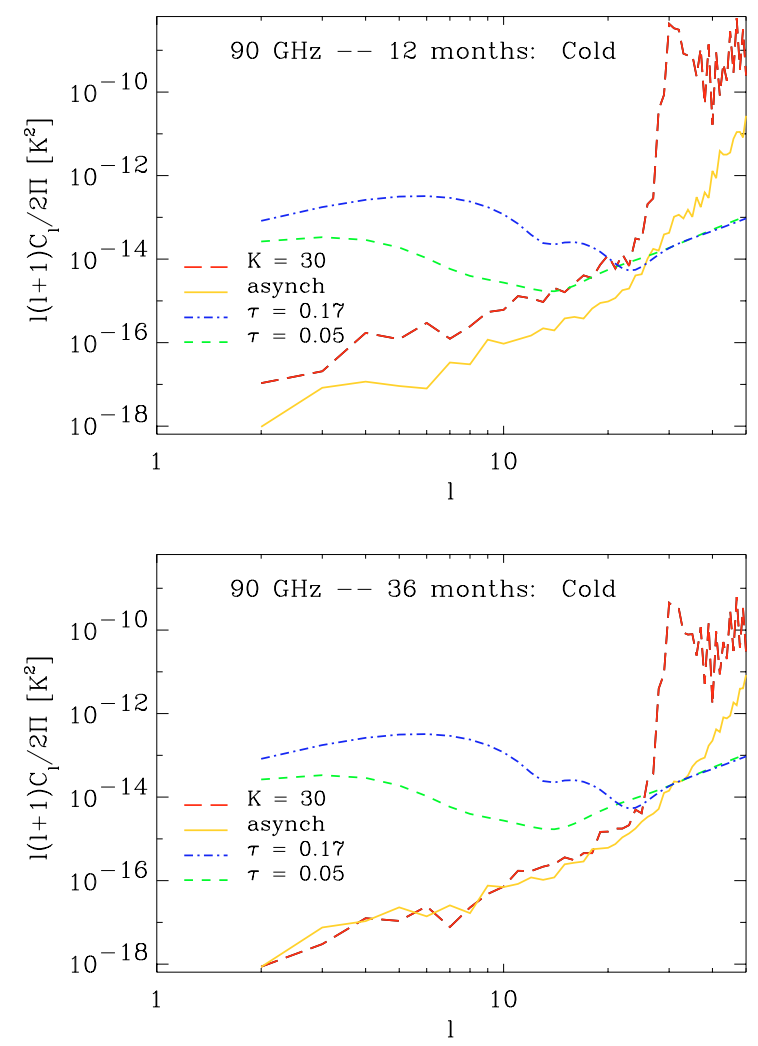

Fig. 6. As Fig. 5 but for the cryo-stage of the $90 \mathrm{GHz}$ receiver.

and the synchronous one with $K=30$, the latter representing the worst condition, especially if all the $0.1 \mathrm{~K}$ variation is considered of such a kind. The spectra are reported in Fig. 6 for two different mission durations. Although higher than that from the horn section, the contamination is again well below the sky signal already for a 12-month mission and synchronous fluctuations with $K=30$. Also this contribution, thus, is unlikely to require any data cleaning. It is worth noting that even the low $\tau=0.05$ model is free from contaminations in the $\ell$-range where most of the cosmological signal resides $(\ell<10)$.

\section{Summary and conclusions}

In this work the importance of the errors induced by thermal instabilities has been evaluated for the SPOrt experiment. In particular we have computed and analyzed

1. The transfer functions of the front-end passive devices (horn, OMT, polarizer) describing how the temperature fluctuations are reflected on the signal. 
2. The dumping effects on the final sky maps due to both the SPOrt scanning strategy and the behaviour of the fluctuations.

The main results can be summarized as follows:

- The correlation scheme of SPOrt has a low sensitivity to temperature instabilities. In fact, the transfer functions are the products between $(A-1)$ terms, common to total power architectures, and $S P_{\text {pol }}$ and $S P_{\text {omt }}$ coefficients, specific to correlators. Thus, these benefit from extra-terms which further reduce the impact of thermal fluctuations, making correlators a suitable solution for measurements of weak signals.

- The 70-day precession of the ISS orbit allows cancellation effects in the case of fluctuations synchronous with the Sun illumination $(K=1)$. The dumping is more than 2 orders of magnitude.

- Fluctuations with shorter periods $(K>1)$ show stronger dumping, but only on angular scales larger than $180^{\circ} / K$. In fact, they move the contamination to multipoles $\ell>\ell_{K} \sim$ $K$, leaving the range $\ell<\ell_{K}$ much cleaner than in the case with $K=1$. As a consequence, the condition $K>30$ is enough to minimize the contamination on the angular scales accessible to SPOrt $(\ell<25)$.

- Fluctuations uncorrelated with the Sun illumination represent the best condition, although their contamination level is not so far from the synchronous case with $K>30$;

We would like to point out that the results for $K=1$ are mainly due to different Sun illumination conditions during the different precession periods. Therefore, in case of no changes in the thermal conditions during different scans, this dumping effect would not be present. An example of such a condition is the Lagrangian point L2 of the Earth-Sun system, where the same pixel is observed every 6 months with similar illumination conditions (Sun in the back of the spacecraft). L2 enjoys an optimal environment stability, much better than that in the low-orbit of ISS. However, no dumping effects due to different Sun illumination conditions can be expected, so that the impact of scan synchronous fluctuations must be carefully evaluated, especially when dealing with the weak CMBP signal. In this case relevant benefits can only be obtained from a proper scanning strategy with several crossing at different angles among the scans, as that of WMAP.

The analysis of the actual antenna system of SPOrt shows a low sensitivity to thermal instabilities, achieved thanks to both the correlation scheme and new devices developed by the SPOrt team to minimize systematic effects. In spite of this, the fluctuations induced by the ISS orbit would be too large to allow a clean detection of CMBP in absence of an active thermal control of the horns. On the other hand, the active control adopted for the horns leads to fluctuations featuring an amplitude within $\pm 0.2 \mathrm{~K}$ (instead of $\pm 3 \mathrm{~K}$ ) and a frequency $f_{\text {tf }}>30 f_{\text {day }}$. Our analysis shows that the resulting contamination is well below the expected cosmological signal, leaving the $E$-mode spectrum of the CMBP uncontaminated.

The thermal fluctuations of the cold-stage generate a contamination low enough to allow again a clean detection of the
CMBP signal, although this is - surprisingly - the most contaminant source, even larger than that of the warm stage.

The cold stage contribution, being much larger than that from the warm section, in practice represents the total contamination for the SPOrt experiment, which thus, in spite of an unfriendly environment, appears to be robust against systematics induced by thermal fluctuations.

Acknowledgements. This work has been carried out in the frame of the SPOrt experiment, a programme of the Italian Space Agency (Agenzia Spaziale Italiana: ASI). We thank Riccardo Tascone for useful discussions and the referee for useful comments. M.Z., C.M. and C.S. acknowledge ASI grants. Some results of this paper have been derived using the HEALPix ${ }^{3}$ (Górski et al. 1999). We acknowledge the use of the CMBFAST package.

\section{Appendix A: Temperature dependence of attenuation, $S \boldsymbol{P}_{\mathrm{pol}}$ and $\boldsymbol{S} \boldsymbol{P}_{\mathrm{omt}}$}

In general, the attenuation $A$ of passive devices like feed horns, polarizers and OMTs dependes on the electric resistivity in a fashion (e.g. see Collin 1996)

$A_{\mathrm{dB}} \propto \sqrt{\rho}$,

where $A_{\mathrm{dB}}$ is expressed in $\mathrm{dB}$ unit. In the temperature range of interest for SPOrt $(80-300 \mathrm{~K})$, the resistivity $\rho$ has a linear behaviour with respect to the temperature $T$ for most of the materials the devices are made of (e.g. Al, Au, Ag), so that

$A_{\mathrm{dB}} \propto \sqrt{T}$

that means the linear attenuation follows

$A=\mathrm{e}^{K_{A} \sqrt{T}}$

where $K_{A}$ is a proper constant. At lower temperature, the linear behaviour of $\rho$ is no more valid and a proper function of $T$ has to be used. However, this dependence weekens ( $\rho$ is going to be constant toward $T=0 \mathrm{~K}$ ) and the variations we derive below can be considered as a worst case estimate.

For devices with low attenuation $(A=1+x$, with $x \ll 1)$ a linear approximation can be applied. In this limit the simple relation

$A \sim 1+K_{A} \sqrt{T}$

holds and the variations with respect to $T$ of the attenuations and the $S P_{\mathrm{pol}}, S P_{\mathrm{omt}}$ coefficients can be easily computed. In particular we have the following results:

1. Attenuations: expanding to the first order of $K_{A} \sqrt{T}$ one gets

$$
\begin{aligned}
\Delta A & =\frac{K_{A} \sqrt{T}}{2 T} A \Delta T \\
& \sim \frac{K_{A} \sqrt{T}}{2 T} \Delta T \\
& \sim \frac{A-1}{2 T} \Delta T .
\end{aligned}
$$

\footnotetext{
${ }^{3}$ http://www. eso.org/science/healpix/
} 
Alternatively, starting from the first row of the previous equation, one can also write

$\Delta A \sim \frac{A-1}{2 T} A \Delta T$.

In this paper we use both of them.

2. $A-1$ : in similar way, it can be written

$\Delta(A-1) \sim \frac{A-1}{2 T} \Delta T$,

or, equivalently,

$\Delta(A-1) \sim \frac{A-1}{2 T} A \Delta T$.

3. $S P_{\text {pol }}$ coefficient: Eq. (3) can be written as

$$
\begin{aligned}
S P_{\mathrm{pol}} & =\frac{1}{2}\left(1-\frac{A_{\|}}{A_{\perp}}\right) \\
& =\frac{1}{2}\left(1-\mathrm{e}^{\left(K_{\|}-K_{\perp}\right) \sqrt{T}}\right) \\
& \sim \frac{1}{2}\left(K_{\perp}-K_{\|}\right) \sqrt{T}
\end{aligned}
$$

where $K_{\perp}$ and $K_{\|}$are the $K_{A}$ coefficients of $A_{\perp}$ and $A_{\|}$, respectively. Therefore, the variation of $S P_{\text {pol }}$ writes

$$
\begin{aligned}
\Delta S P_{\mathrm{pol}} & \sim \frac{1}{2}\left(K_{\perp}-K_{\|}\right) \sqrt{T} \frac{\Delta T}{2 T} \\
& \sim \frac{S P_{\mathrm{pol}}}{2 T} \Delta T .
\end{aligned}
$$

4. $S P_{\text {omt }}$ coefficient: this contains not only attenuation terms, but also the isolations $S_{A 2}, S_{B 1}$ between the two OMT arms. The latter mainly depends on the geometry of the device rather than on resistive effects, so that, as a first approximation, they can be considered constant with respect to the temperature. Equation (2) can be written as

$S P_{\mathrm{omt}}=S P_{\mathrm{omt}}^{A}+S P_{\mathrm{omt}}^{B}$,

with

$S P_{\mathrm{omt}}^{A}=A_{\mathrm{omt}} S_{A 1} S_{B 1}^{*}$,

$S P_{\text {omt }}^{B}=A_{\text {omt }} S_{A 2} S_{B 2}^{*}$.

Reminding that the attenuations along the OMT arms are given by $A_{\mathrm{omt}}^{A}=1 /\left|S_{A 1}\right|^{2}, A_{\mathrm{omt}}^{B}=1 /\left|S_{B 2}\right|^{2}$, one can write

$$
\begin{aligned}
\Delta S P_{\mathrm{omt}}^{A} \sim & \frac{A_{\mathrm{omt}}-1}{2 T} A_{\mathrm{omt}} S_{A 1} S_{B 1}^{*} \Delta T \\
& -\frac{A_{\mathrm{omt}}^{A}-1}{4 T} A_{\mathrm{omt}} S_{A 1} S_{B 1}^{*} \Delta T \\
= & {\left[\left(A_{\mathrm{omt}}-1\right)-\frac{1}{2}\left(A_{\mathrm{omt}}^{A}-1\right)\right] \frac{S P_{\mathrm{omt}}^{A}}{2 T} \Delta T . }
\end{aligned}
$$

The OMT attenuation $A_{\text {omt }}$ is the average of those along the two arms, for which, in general, the relation $A_{\mathrm{omt}}^{A} \sim A_{\mathrm{omt}}^{B} \sim$ $A_{\text {omt }}$ holds. Thus, we can write

$\Delta S P_{\mathrm{omt}}^{A} \sim \frac{A_{\mathrm{omt}}-1}{4 T} S P_{\mathrm{omt}}^{A} \Delta T$.

A similar relation holds for the other term

$\Delta S P_{\mathrm{omt}}^{B} \sim \frac{A_{\mathrm{omt}}-1}{4 T} S P_{\mathrm{omt}}^{B} \Delta T$

so that the variation of $S P_{\mathrm{omt}}$ is given by

$$
\begin{aligned}
\Delta S P_{\mathrm{omt}} & =\Delta S P_{\mathrm{omt}}^{A}+\Delta S P_{\mathrm{omt}}^{B} \\
& \sim \frac{A_{\mathrm{omt}}-1}{4 T} S P_{\mathrm{omt}} \Delta T .
\end{aligned}
$$

\section{References}

Carretti, E., Tascone, R., Cortiglioni, S., Monari, J., \& Orsini, M. 2001, New Astron., 6, 173

Carretti, E., Cortiglioni, S., Sbarra, C., \& Tascone, R. 2004, A\&A, 420, 437

Collin, R. E. 1996, Foundation for Microwave Engineering (McGrawHill)

Cortiglioni, S., Bernardi, G., Carretti, E., et al. 2003, Proc. of the 16th ESA Symp. on European Rocket and Balloon Programmes and Related Research, ESA Proc., SP-530, 271

Cortiglioni, S., Bernardi, G., Carretti, E., et al. 2004, New Astron., 9, 297

Franco, G., Fosalba, P., \& Tauber, J. A. 2003, A\&A, 405, 349

Górski, K. M., Hivon, E., \& Wandelt, B. D. 1999, in Proc. of the MPA/ESO Cosmology Conference Evolution of Large-Scale Structure, ed. A. J. Banday, R. S. Sheth, \& L. da Costa, Print Partners Ipskamp, NL, 37 [arXiv: astro-ph/9812350]

Hu, W., Hedman, M. M., \& Zaldarriaga, M. 2003, PRD, 67, id. 043004

Kaplan, J., \& Delabrouille, J. 2002, Astrophysical Polarized Backgrounds, ed. S. Cecchini, S. Cortiglioni, R. Sault, \& C. Sbarra, AIP Conf. Proc., 609, 209

Kogut, A., Spergel, D. N., Barnes, C., et al. 2003, ApJS, 148, 161

Kraus, J. D. 1986, Radio Astronomy (Cygnus-Quasar Books)

Leahy, J. P., Yurchenko, V., Hastie, M. A., Bersanelli, M., \& Mandolesi, N. 2002, Astrophysical Polarized Backgrounds, ed. S. Cecchini, S. Cortiglioni, R. Sault, \& C. Sbarra, AIP Conf. Proc., 609,215

Mennella, A., Bersanelli, M., Burigana, C., et al. 2002, A\&A, 384, 736

Page, L., Jackson, C., Barnes, C., et al. 2003, ApJ, 585, 566

Peverini, O. A., Tascone, R., Baralis, M., et al. 2003, in International Conference on Electromagnetics in Advanced Applications ICEAA '03, Torino 8-12 September 2003, Proceedings of ICEAA '03, 363

Piat, M., et al. 2000, Proceedings of LTD-9, NIMA, 444, 413

Sbarra, C., Carretti, E., Cortiglioni, S., et al. 2003, A\&A, 401, 1215

Spergel, D. N., Verde, L., Peiris, H. V., et al. 2003, ApJS, 148, 175 